\title{
Performance evaluation of an efficient five input majority gate design in QCA nanotechnology
}

\author{
Amanpreet Sandhu, Sheifali Gupta \\ Department of Electronics and Communication Engineering, Chitkara University Institute of Engineering and \\ Technology Chitkara University, India
}

\begin{tabular}{l}
\hline Article Info \\
\hline Article history: \\
Received Jul 9, 2019 \\
Revised Sep 20, 2019 \\
Accepted Oct 11, 2019 \\
\hline
\end{tabular}

Keywords:

Full adder

Majority gate

QCADesigner

QCAPro

QCA

\begin{abstract}
Quantum-dot-cellular-automata (QCA) is the imminent transistor less technology, considered at nano level with high speed of operation and lower power dissipation features. The present paper proposes a novel and an efficient 5-input coplanar majority gate (PMG) with improved structural and energy efficiency. The proposed gate consumes an occupational area of $0.01 \mu \mathrm{m}^{2}$ with 17 QCA cells which is $50 \%$ less in comparison to the best designs reported in literature. The proposed structure is also more energy efficient because it dissipates $21.1 \%$ less energy than the best reported designs. The correctness of a proposed majority gate is verified by designing a single bit full adder. The new 1-bit full adder design is structural efficient and robust in terms of gate count and clock delay. It consumes occupational area of $0.05 \mu \mathrm{m}^{2}$ with 58 QCA cells showing $16.6 \%$ improvement in structural efficiency as compared to the best design reported in. It is having a gate count of 4 with the delay of 1 clock cycle. Here, the QCADesigner and QCAPro tools are utilized for the simulation and energy dissipation analysis of proposed majority gate and full adder design.
\end{abstract}

Copyright $@ 2019$ Institute of Advanced Engineering and Science. All rights reserved.

\section{Corresponding Author:}

Amanpreet Sandhu,

Department of Electronics and Communication Engineering,

Chitkara University Institute of Engineering and Technology Chitkara University, India.

Email: amanpreet.sandhu@ chitkara.edu.in

\section{INTRODUCTION}

Designing and fabricating complementary metal oxide semiconductor (CMOS) based logic devices at nano scale [1] has an issues like oxide thickness, thermal reliability and power dissipation [2]. Hence the industries are in search of new techniques which could aid the scaling of CMOS. Researchers are well aware that the CMOS technology could be continued only for a decade. Some of the alternate technologies like QCA, single electron tunneling (SET), carbon nanotubes (CNT) came into existence. QCA is one of the competitive alternate technology [3] that has none of the above said problems. The benefit of QCA devices over regular CMOS circuits are the absence of electron flow for charge transfer and absence of metallic interconnects which are the main source of IR losses with low power consumption [4]. Hence QCA [5] is more prudent than CMOS technology.

Many QCA logic designs have been implemented during recent years. Various 5-input majority gates [6-15], 1-bit full adder designs [6-8, 10, 12, 14, 16, 17-29], multiplier designs [30-33], RAM cell structures [34-35], flip flops [36-39] and logic circuits [40, 41]. In the above work, most of the circuits were not potent and hence susceptible to various defects at fabrication level because of the wire-crossing structures of QCA cells. Here, an effective use of cross overs can reduce the number of QCA cells, complexity and total cost. Multiple cross over wire designs results in various fabrication defects [42] and area overhead. One can replace these multilayer structures with 45 degree rotated cells which results in coplanar cross over designs. Such coplanar cross over designs are utilizing two types 
of QCA cells which results in a problem of increased fabrication cost and reduced robustness [43]. Hence there is a need to design robust single layer QCA structures which uses only single type QCA cells (that is 90 degree cells). The basic idea is to propose a design of a robust and energy efficient 5-input majority gate and investigate the energy dissipation of existing majority gate with PMG. The PMG proposed in this paper is utilizing lesser area with reduced power dissipation as compared to the best designs reported in the literature. The correctness of PMG is validated by designing a one bit full adder based on proposed gate.

In this work, a coplanar 5-input novel and efficient majority gate is proposed. To measure its effictiveness, 1-bit novel full adder structure is designed using PMG. The remaining paper is arranged as follows. Section 2 showcase the basic concept of QCA structure and the clocking concepts. Section 3 describes the existing QCA based five input majority gates. Section 4 describes a proposed 5-input novel majority gate design, simulation results, its physical proof and energy dissipation analysis. The energy dissipation analysis is done using QCAPro tool. Section 5 represents a new robust full adder circuit design using the PMG, its simulation, energy dissipation analysis and comparison of proposed robust full adder circuit with the existing designs in terms of area and delay. Section 6 compares the result of PMG with the existing designs in terms of occupational area and interference. The Conclusion is presented in section 7.

\section{REVIEW OF A QCA CELL}

QCA cell is the fundamental nano structure which can construct all elements of a circuit (wiring and computing). A basic QCA cell is having four quantum dots placed at the extreme edges of a quantum cell. Out of which, two quantum dots contain free electrons in a diagonal direction. These two electrons can exchange their positions by lowering the barrier potential between them to achieve $\mathrm{P}=+1$ (logic 0 ) or $\mathrm{P}=-1$ (logic 1) polarization state [16] as depicted in Figure 1. These two free electrons confines with in a QCA cell and can never tunnel between the adjacent QCA cells. Hence when array of QCA cells placed adjacent to one another to form a wire, only a polarization state (columbic charge) will travel along the wire. Such an array of QCA cells can be used to construct wire or any logic structure. Hence there is less power dissipation because of the change in the polarisation and propagation of columbic charge (absence of flow of electrons). Therefore a QCA technology can be an alternative to that of a CMOS technology. The digital structure in QCA is designed by joining these cells in cascade.

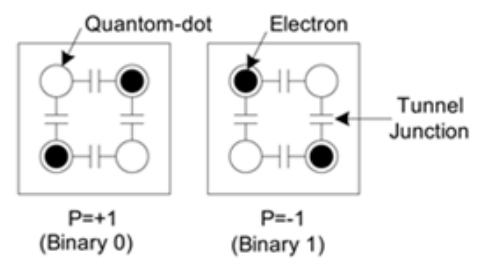

Figure 1. QCA cells with two polarization states

\subsection{Basic structures}

By connecting basic QCA cells in cascade, a wire can be formed shown in Figure 2(a). Other QCA structures like inverter and majority gate of three inputs can also be constructed using these quantum cells. An inverter circuit of Figure 2(b) invert its state because the output cell is in the diagonal orientation (interaction) with respect to the adjacent QCA cell. A majority gate works on the principle that the value of the output cell is true if majority of the input QCA cells are true. The QCA structure of a3-input majority gate is illustrated in Figure 2(c). This gate can be further configured to form AND and OR gate structures. The function of 3-input majority gate is exhibited by the following equation:

$$
\mathrm{M}(\mathrm{A}, \mathrm{B}, \mathrm{C})=\mathrm{AB}+\mathrm{BC}+\mathrm{AC}
$$

The majority gate with 5-inputs based designs are much faster and are having less area as compared to the same designs made using the majority gate with 3-inputs. Figure 2(d) represents a basic structure of 5-input majority gate. Its Boolean function is described (2)

$$
\mathrm{MG}(\mathrm{A}, \mathrm{B}, \mathrm{C}, \mathrm{D}, \mathrm{E})=\mathrm{ABC}+\mathrm{ABD}+\mathrm{ABE}+\mathrm{ACD}+\mathrm{ACE}+\mathrm{ADE}+\mathrm{BCD}+\mathrm{BCE}+\mathrm{BDE}+\mathrm{CDE}
$$




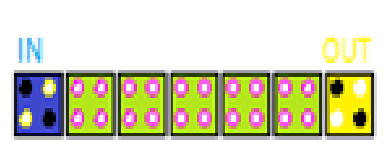

(a)

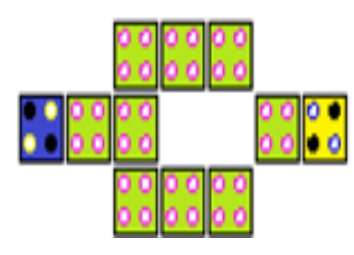

(b)

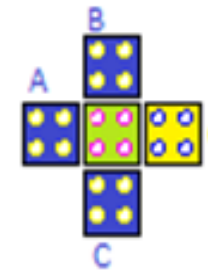

(c)

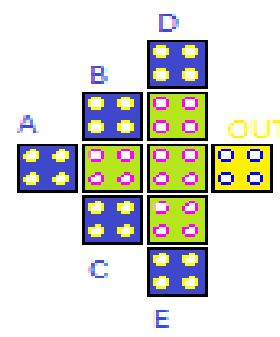

(d)

Figure 2. (a) QCA Wire, (b) Inverter gate, (c) 3-Input Majority gate, (d) 5-input Majority gate

\subsection{Clocking}

In switch phase, the barrier potential of a QCA cell starts increasing, hence moving from unpolarized state to polarized state. In this state, the polarization state of the QCA cell will depend upon its neighboring cells. In Hold phase, the barrier potential will remain constant and the QCA cell is completely polarized. Now it becomes independent of its neighboring cells. In release phase, the barrier potential of QCA cell starts reducing, hence moving from polarized state to unpolarized state. In relax phase, the barrier potential of the QCA cell becomes zero and hence the cell will be unpolarized.

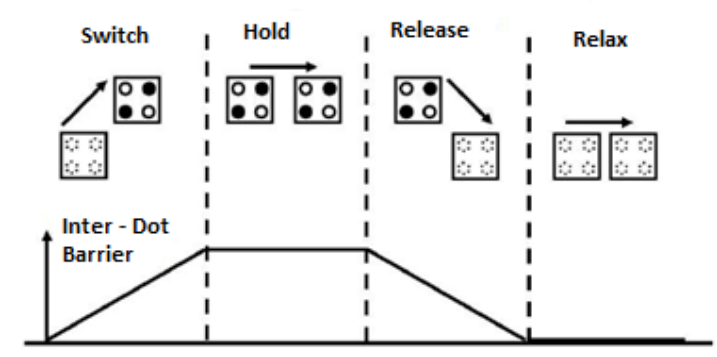

Figure 3. QCA clocking with four phases

\section{EXISTING 5-INPUT MAJORITY GATES BASED ON QCA}

In addition to 3-input majority gate design many researchers tried to implement the digital circuits using 5-Input majority gate. The purpose of designing digital circuits using 5-input Majority gate is reduced area, latency, faster speed of operation than the traditional designs. Various five input majority gates existing in the literature are described in this section. Many digital circuits implementing 5-Input Majority gate using (2) have been designed in [6, 8, 19, 22, 24].

The design [6] shown in Figure 4(a), is utilizing only 10 QCA cells with an area of $0.01 \mu \mathrm{m}^{2}$. This 5-input majority gate is having a drawback that its output QCA cell is trapped by the input QCA cells, hence limited the access of output cell in a single layer only. The majority gate design [8] Figure 4(b) also utilizing 10 QCA cells with an area of $0.01 \mu \mathrm{m}^{2}$ but it causes an interference between the input QCA cells as they are too close to each other. However the design presented in [22] depicted in Figure 4(c), tries to overcome the previous disadvantages but at the cost of increased area from $0.01 \mu \mathrm{m}^{2}$ to $0.02 \mu \mathrm{m}^{2}$. The designs in [19] and [24] Figure 4(d) and Figure 4(e) also shows the improvement in terms of no interference and accessibility to single as well as multi-layer designs. These designs are not so encouraging because of their increased up to $0.03 \mu \mathrm{m}^{2}$. The design in [19] is utilizing 42 QCA cells whereas the design in are using 51 QCA cells [24]. 


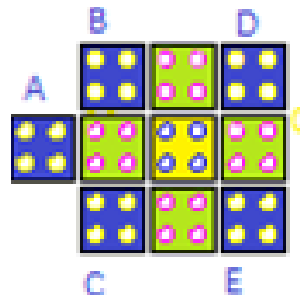

(a)

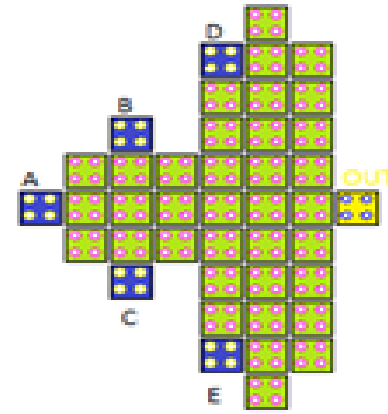

(d)

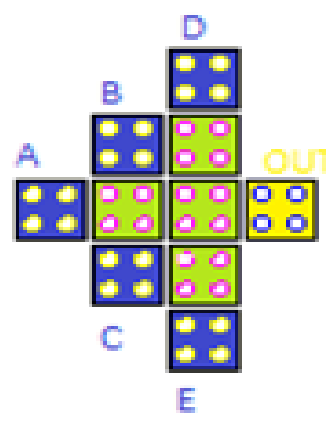

(b)

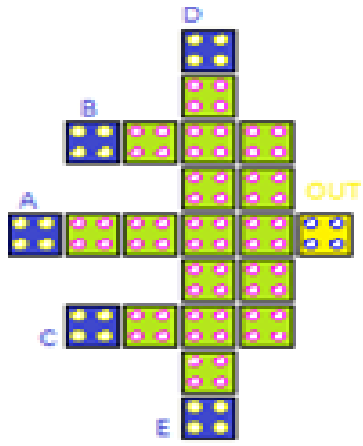

(c)

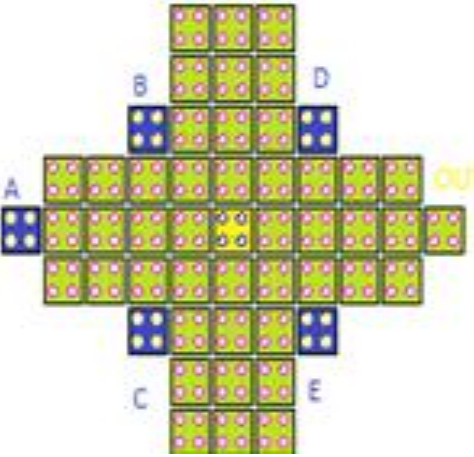

(e)

Figure 4. Various 5-input majority gates (a-e) design

\section{PROPOSED FIVE-INPUT MAJORITY GATE (PMG)}

\subsection{Structural analysis}

The five input novel majority gate proposed in this paper is utilizing 17 QCA cells having an area of $0.01 \mu \mathrm{m}^{2}$ as shown in Figure 5(a). It has no interference between the input cells and can be used for both single layer as well as multi-layer design. Figure 5(b) shows the simulation waveform for the PMG. From the simulation results, it is clear that when majority number of inputs are at high logic then the output is also at logic high.

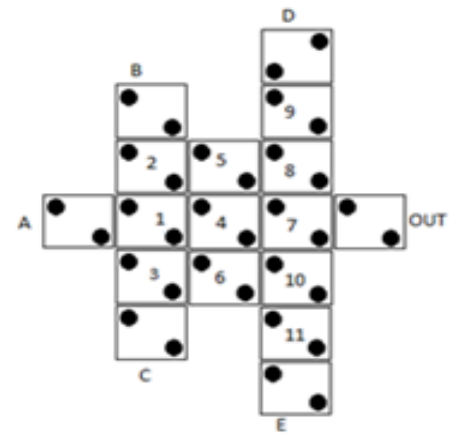

(a) 


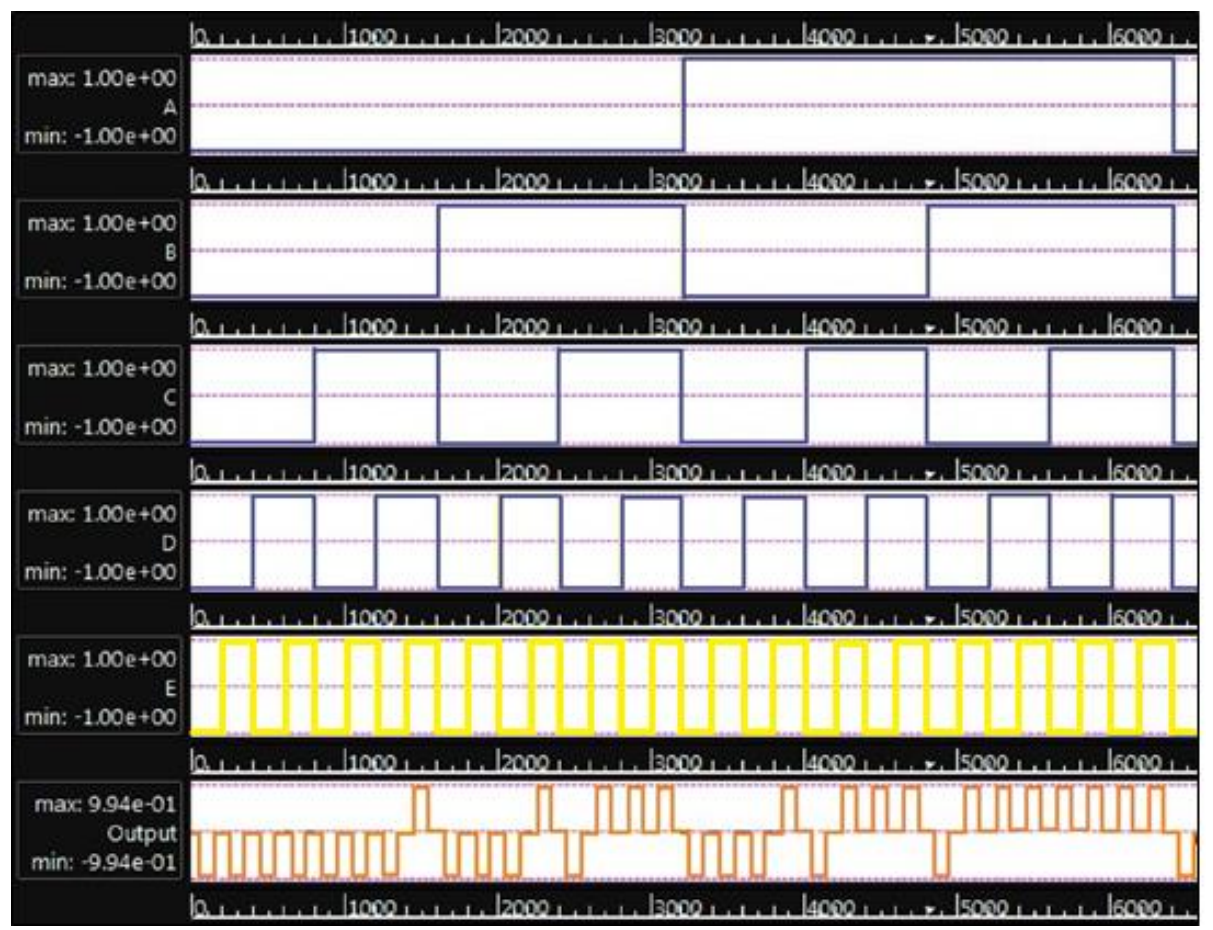

(b)

Figure 5. The Proposed five input majority gate, (a) Structure, (b) Simulation results

\subsection{Physical proof}

The proposed 5-input coplanar majority gate (PMG) has $2^{5}=32$ input combinations. Accuracy of these 32 input combinations need to be verified but due to insufficient space, only one state is proven for consideration. Rest of the other states can be similarly verified. All the QCA cells of PMG are of equal size $(18 \mathrm{~nm} * 18 \mathrm{~nm})$ and are separated from each other by $2 \mathrm{~nm}$ space. The electrons in each cell are positioned in a manner that they must acquire minimum potential energy to become stable. The lesser the potential energy of a qca cell, the more stable it is. The potential energy between two electron charges in a QCA cell can be calculated as:

$$
U=\frac{k q_{1} q_{2}}{r}=\frac{A}{r}
$$

Where $A=k q_{1} q_{2}=9 * 10^{9} *(1.6)^{2} * 10^{-38}=23.04 * 10^{-29}$

Where, $\mathrm{U}=$ Potential Energy, $\mathrm{K}=$ Coulomb' $\mathrm{s}$ law Constant, $\mathrm{r}=$ distance between two electrons and $\mathrm{q}_{1}$ and $\mathrm{q}_{2}$ are charges of electrons.

$$
U_{T}=\sum_{i=1}^{n} U_{i}
$$

Where $\mathrm{U}_{\mathrm{T}}=$ Summation of potential energies [34, 44].

In this section, the stability analysis of proposed $\mathrm{PMG}$ is done on one state that is $\mathrm{A}=\mathrm{B}=\mathrm{C}=\mathrm{E}=1$ and $\mathrm{D}=0$, by finding the potential energy between Input cells (i.e. $\mathrm{A}, \mathrm{B}, \mathrm{C}, \mathrm{D}$ and $\mathrm{E}$ ) and their corresponding middle cells (I.e. cell no. 1, 2, 3, 9 and 11). Figures 6(a,b) shows Cell 1 with electrons $\mathrm{x}$ and $\mathrm{y}$ in two different states. Next step is to find which state is more stable by calculating their potential energies separately. 


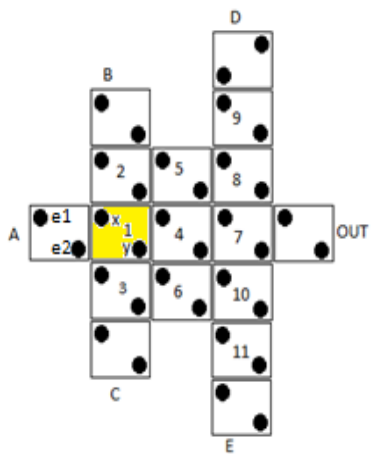

(a)

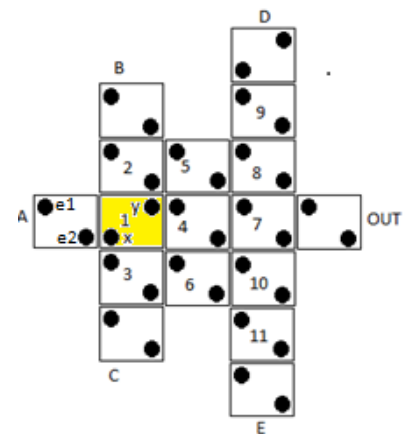

(b)

Figure 6. Two states of cell 1,(a) Cell 1=Logic "1", (b) Cell 1=logic “ 0 "

Firstly the potential energy of cell 1(highlighted with yellow colour) in Figure 6(a) will be calculated, when the value of cell 1 is logic ' 1 '. The polarization of cell 1 is affected by input cell A only because it is the only adjacent input cell for cell 1.The potential energy of electron $x\left(U_{x}\right)$ is calculated with reference to electron e 1 and $\mathrm{e} 2$ called as $\mathrm{U}_{\mathrm{x} 1}$ and $\mathrm{U}_{\mathrm{x} 2}$. In the simillar way potential energy of electron $\mathrm{y}\left(\mathrm{U}_{\mathrm{y}}\right)$ is calculated withreference to electron e 1 and $\mathrm{e} 2$ called as $\mathrm{U}_{\mathrm{y} 1}$ and $\mathrm{U}_{\mathrm{y} 2}$. The calculation of potential energies $\mathrm{U}_{\mathrm{x} 1}, \mathrm{U}_{\mathrm{x} 2}, \mathrm{U}_{\mathrm{y} 1}$ and $\mathrm{U}_{\mathrm{y} 2}$ are given below:

$$
\begin{array}{cc}
U_{x 1}=\frac{A}{r 1}=\frac{23.04 * 10^{-29}}{20} \approx 1.152 * 10^{-20}(\mathrm{~J}) & U_{y 1}=\frac{A}{r 1}=\frac{23.04 * 10^{-29}}{42.04} \approx 0.5479 * 10^{-20}(\mathrm{~J}) \\
U_{x 2}=\frac{A}{r 1}=\frac{23.04 * 10^{-29}}{18.11} \approx 0.07024 * 10^{-20}(\mathrm{~J}) & U y_{2}=\frac{A}{r 1}=\frac{23.04 * 10^{-29}}{20} \approx 1.152 * 10^{-20}(\mathrm{~J}) \\
U_{x}=\sum_{i=1}^{2} U_{x i}=1.222 * 10^{-20}(\mathrm{~J}) & U_{y}=\sum_{i=1}^{2} U_{y i}=1.6999 * 10^{-20}(\mathrm{~J}) \\
U_{T 11}=U_{x}+U_{y}=2.92219 * 10^{-20}(\mathrm{~J}) &
\end{array}
$$

Where, $\mathrm{UT}_{11}$ is the total potential energy of cell1 when it is at the ' 1 ' state.

Similarly the results of $\mathrm{U}_{\mathrm{x} 1}, \mathrm{U}_{\mathrm{x} 2}, \mathrm{U}_{\mathrm{y} 1}$ and $\mathrm{U}_{\mathrm{y} 2}$ are calculated when the value of cell 1 is at logic ' 0 ' as shown in Figure 6(b).

$$
U_{x}=\sum_{i=1}^{2} U_{i}=12.376 * 10^{-20}(\mathrm{~J}) \quad U_{y}=\sum_{i=1}^{2} U_{i}=1.46258 * 10^{-20}(\mathrm{~J})
$$

Where, $\mathrm{UT}_{10}$ is the total potential energy of cell1 when it is at the ' 0 ' state. The results shows that the potential energy of $\mathrm{UT}_{11}$ is lower than the $\mathrm{UT}_{10}$. So the Cell1 will acquire the polarization state of ' 1 ' because it achieves lower potential energy with more stability.

In the same way the potential energies of other middle cells which are adjacent to input cells will be calculated by assuming them to be at the state " 1 " and " 0 " respectively. The final results of potential energies for adjacent middle cells $(2,3,9$, and 11) are given below.

Cell 2: $\quad$ At Logic “ $1 " U_{T 21}=4.1239 * 10^{-20}(j)$

$$
\text { At Logic " } 0 " U_{T 20}=13.8387 * 10^{-20}(j)
$$

Cell 3: $\quad$ At Logic " $1 " U_{T 31}=4.1241 * 10^{-20}(j)$

At Logic “ 0 " $U_{T 30}=13.8387 * 10^{-20}(j)$

Cell 9: $\quad$ At Logic " $1 " U_{T 91}=13.8387 * 10^{-20}(j)$

At Logic " 0 " $U_{T 90}=4.1240 * 10^{-20}(j)$

Cell 11: $\quad$ At Logic " 1 " $U_{T 111}=4.1241 * 10^{-20}(j)$

At Logic " $0 " U_{T 110}=13.8387 * 10^{-20}(j)$ 
From the above results, it is clear that when the inputs are $\mathrm{A}=\mathrm{B}=\mathrm{C}=\mathrm{E}=1$ and $\mathrm{D}=0$, then cell 2 , Cell 3 and Cell 11 will remain at the logic 1 because potential energies $\mathrm{UT}_{21}, \mathrm{UT}_{31}, \mathrm{UT}_{111}$ are less than $\mathrm{UT}_{20}, \mathrm{UT}_{30}$ and $\mathrm{UT}_{110}$ respectively. Whereas Cell 9 will be at the logic 0 because $\mathrm{UT}_{90}$ is lesser than $\mathrm{UT}_{91}$. This is practically true also because its adjacent input Cell D is at logic 0 . By considering the achieved results, the proposed QCA structure for implementing 5-input novel majority gate is fully correct and results in a precise output.

\subsection{Energy dissipation analysis}

Low power dissipation, even below traditional $K T$, is one of the main feature of QCA nanotechnology. QCAPro is one of the accurate power estimation tool which uses non-adiabatic power dissipation model to estimate the switching power loss in QCA [44]. The basics of this model was taken from quasi adiabatic model in [23]. According to this model, the expectation energy value of cell for every clock cycle is described as:

$$
E=\frac{\hbar}{2} \cdot \vec{\Gamma} \cdot \vec{\lambda}
$$

Where, $\vec{\lambda}=$ coherence vector $\vec{\Gamma}=3 \mathrm{D}$ energy vector

Now, the total power of single QCA cell at any instant is:

$$
P_{\text {total }}=\frac{d E}{d t}=\frac{d}{d t}\left[\frac{\hbar}{2} \cdot \vec{\Gamma} \cdot \vec{\lambda}\right]=\frac{\hbar}{2}\left[\frac{d \vec{\Gamma}}{d t} \cdot \vec{\lambda}\right]+\frac{\hbar}{2}\left[\vec{\Gamma} \cdot \frac{d \vec{\lambda}}{d t}\right]=P_{1}+P_{2}
$$

The first term i.e. $P_{1}=\frac{\hbar}{2}\left[\frac{d \vec{\Gamma}}{d t} \cdot \vec{\lambda}\right]$ indicates two components, first is transfer of power from clock signal to the QCA cell and the second is power gain due to the difference in input and output signal power.

The second term $P_{2}=\frac{\hbar}{2}\left[\vec{\Gamma} \cdot \frac{d \vec{\lambda}}{d t}\right]$ indicates instantaneous power dissipation. In one clock cycle, the energy dissipation in a QCA cell is calculated by integrating $P_{2}$ over time. Therefore,

$$
\begin{aligned}
& E_{\text {diss }}=\int_{-T}^{T} P_{2} d t=\frac{\hbar}{2} \int_{-T}^{T} \vec{\Gamma} \cdot \frac{d \vec{\lambda}}{d t} d t \\
& E_{\text {diss }}=\frac{\hbar}{2}\left([\vec{\Gamma} \cdot \vec{\lambda}]_{-T}^{T}-\int_{-T}^{T} \vec{\lambda} \cdot \frac{d \vec{\Gamma}}{d t} d t\right)
\end{aligned}
$$

The value of energy dissipation is maximum for maximum changing rate of $\vec{\Gamma}$. So the upper bound power dissipation is given by:

$$
P_{\text {diss }}=\frac{E_{\text {diss }}}{T_{c c}}\left\langle\frac{\hbar}{2 T_{c c}} \vec{\Gamma}_{+} \times\left[-\frac{\vec{\Gamma}_{+}}{\left|\vec{\Gamma}_{+}\right|} \tan h\left(\frac{\hbar\left|\vec{\Gamma}_{+}\right|}{k_{B} T}\right)+\frac{\vec{\Gamma}_{-}}{\left|\vec{\Gamma}_{-}\right|} \tan h\left(\frac{\hbar\left|\vec{\Gamma}_{-}\right|}{k_{B} T}\right)\right]\right\rangle
$$

Here, $k_{B}$ represents Boltzmann Constant, T represents temperature. The author of [33] has presented a power dissipation model in which total power is classified as leakage power and switching power. Here leakage power is a power loss at clock transitions at leading edge or trailing edge of the pulse and switching power loss is due to the switching state of the cell. Based upon this, a tool called QCAPro is developed which estimates average power dissipation of the circuit.

In this paper, the energy dissipation of proposed 5-input coplanar majority gate is analyzed. For this switching energy, leakage energy and total energy dissipation is calculated for three different tunneling energies Table 1 at temperature $\mathrm{T}=2 \mathrm{~K}$. Also the results of proposed 5-input coplanar majority gate is compared with the existing structures proposed in $[6,8,19,22,24]$. Table 1 presents energy dissipation analysis of PMG and the previous existing designs. The comparative results of leakage, switching and total energy dissipation are also illustrated in Figures $7(\mathrm{a}, \mathrm{b}, \mathrm{c})$ respectively. The results calculated in Table 1 concludes that the proposed 5-input coplanar majority gate design has $20.1 \%$ less switching energy, $10.5 \%$ less leakage energy and $21.1 \%$ less total energy dissipation than the best conferred design in [25] for single layer as well as multi-layer design at 1.0Ek tunneling energy level. The existing designs in $[6,8]$ cannot be used for single layer design. So in the graph Figure 7 our results are compared only with the designs that can be used for single layer as well as multilayer structures. Figure 8 shows the thermal layout of proposed five input majority gate at temperature $2.0 \mathrm{k}$ with tunneling energy of $0.5 \mathrm{ek}$. In this, the darker QCA cells indicate more average energy dissipation where as white cells represents the inputs. 
Table 1. Energy dissipation analysis of proposed 5-input coplanar majority gate (PMG) and previous designs

\begin{tabular}{|c|c|c|c|c|c|c|c|c|c|}
\hline \multirow{2}{*}{$\begin{array}{c}5 \text { input } \\
\text { Majority Gate } \\
\text { design }\end{array}$} & \multicolumn{3}{|c|}{$\begin{array}{l}\text { Average leakage Energy } \\
\text { Dissipation }(\mathrm{meV})\end{array}$} & \multicolumn{3}{|c|}{$\begin{array}{l}\text { Average Switching Energy } \\
\text { Dissipation }(\mathrm{meV})\end{array}$} & \multicolumn{3}{|c|}{$\begin{array}{c}\text { Average Energy Dissipation of the } \\
\text { circuit (meV) }\end{array}$} \\
\hline & $0.5 \mathrm{E}_{\mathrm{k}}$ & $1.0 \mathrm{E}_{\mathrm{k}}$ & $1.5 \mathrm{E}_{\mathrm{k}}$ & $0.5 \mathrm{E}_{\mathrm{k}}$ & $1.0 \mathrm{E}_{\mathrm{k}}$ & $1.5 \mathrm{E}_{\mathrm{k}}$ & $0.5 \mathrm{E}_{\mathrm{k}}$ & $1.0 \mathrm{E}_{\mathrm{k}}$ & $1.5 \mathrm{E}_{\mathrm{k}}$ \\
\hline & 1.28 & 4.14 & 7.69 & 11.53 & 10.37 & 9.16 & 12.81 & 14.51 & 16.85 \\
\hline & 1.35 & 4.25 & 7.8 & 10.94 & 9.84 & 8.7 & 12.29 & 14.09 & 16.5 \\
\hline & 4.4 & 11.33 & 24.2 & 35.78 & 32.8 & 29.64 & 40.18 & 46.09 & 53.84 \\
\hline & 8.2 & 26.14 & 49.15 & 117.57 & 110.66 & 102.67 & 125.77 & 136.8 & 151.82 \\
\hline & 6.33 & 17.34 & 38.22 & 92.44 & 86.45 & 76.44 & 98.77 & 103.79 & 114.66 \\
\hline PMG & 3.32 & 10.14 & 18.4 & 28.74 & 26.19 & 23.57 & 32.06 & 36.33 & 41.98 \\
\hline
\end{tabular}

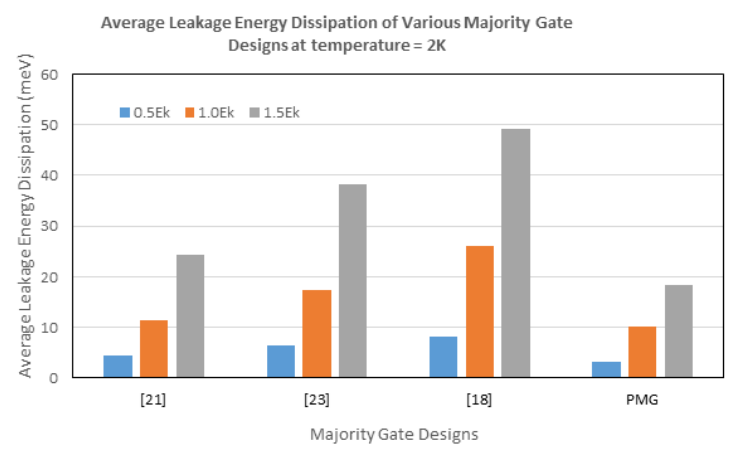

(a)

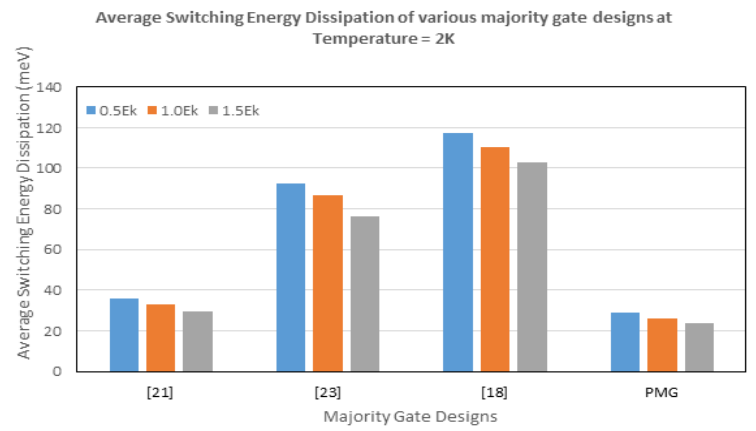

(b)

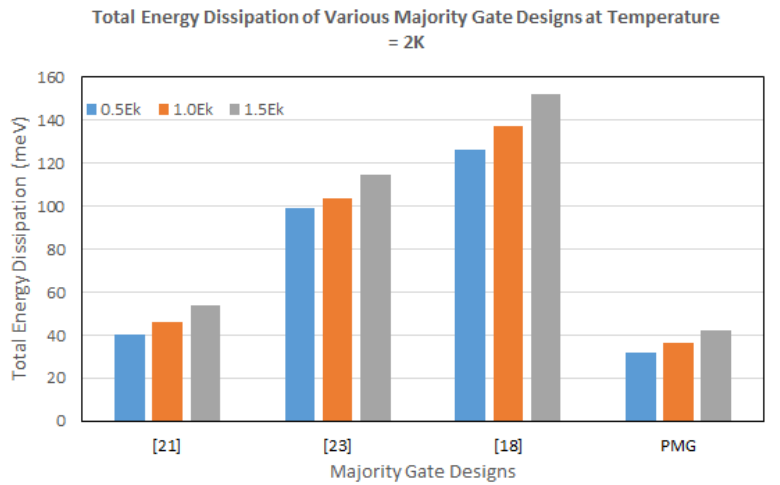

(c)

Figure 7. (a) Leakage energy dissipation, (b) Switching energy dissipation and, (c) Total energy dissipation of the PMG at three different tunneling energies (at $\mathrm{T}=2.0 \mathrm{k}$ )

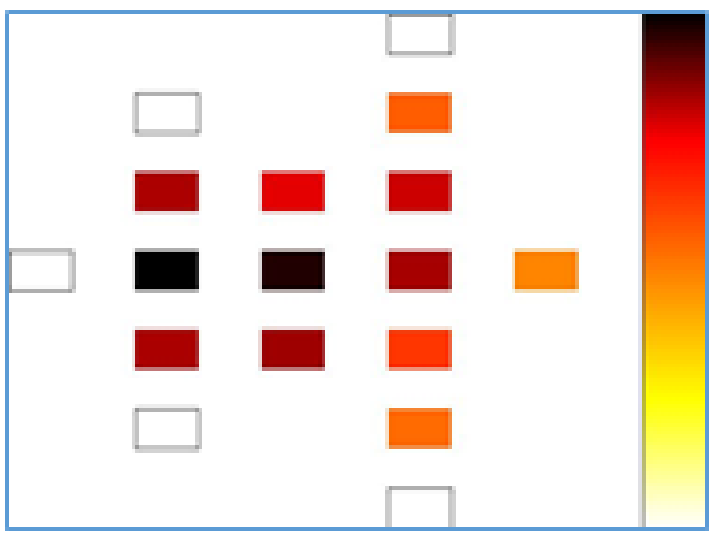

Figur 8. Thermal layout of PMG at temperature $2 \mathrm{~K}$ (Tunneling energy $=0.5 \mathrm{ek}$ ) 


\section{SINGLE BIT QCA FULL ADDER}

\subsection{Proposed 1-bit full adder}

The correctness of proposed coplanar majority gate (PMG) is validated by designing a full Adder structure using proposed gate. The structure of proposed full adder (PFA) using PMG is shown in Figure 9(a). Its QCA equivalent and simulations are represented in Figurs 9(b, c). The PFA circuit adds the two input bits A and B and carry C. The output is taken from SUM and CARRY bits. The PFA structure outperforms the previous designs with 58 QCA cells, occupational area of $0.05 \mu \mathrm{m}^{2}$, gate count of 4 and input to output delay of 1 clock cycle. Its energy dissipation analysis is also done using QCAPro tool and its results are shown in Table 2. The PFA dissipates $129.97 \mathrm{meV}$ energy at $0.5 \mathrm{E}_{\mathrm{k}}$ tunneling energy, $154.73 \mathrm{meV}$ energy at $1.0 \mathrm{E}_{\mathrm{k}}$ tunneling energy and $186.61 \mathrm{meV}$ energy at $1.5 \mathrm{E}_{\mathrm{k}}$ tunneling energy at the temperature of $2 \mathrm{~K}$.

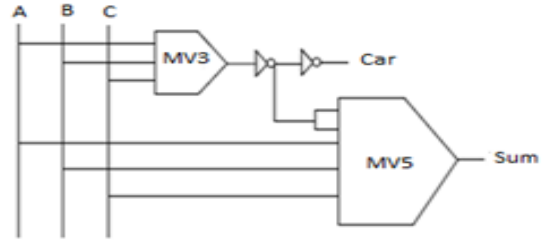

(a)

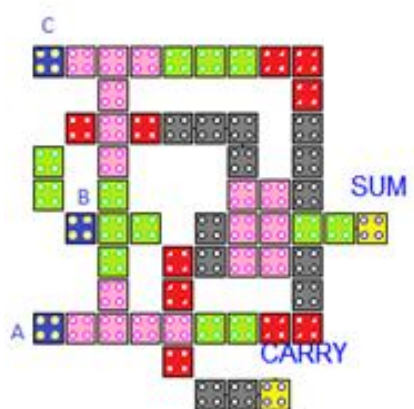

(b)

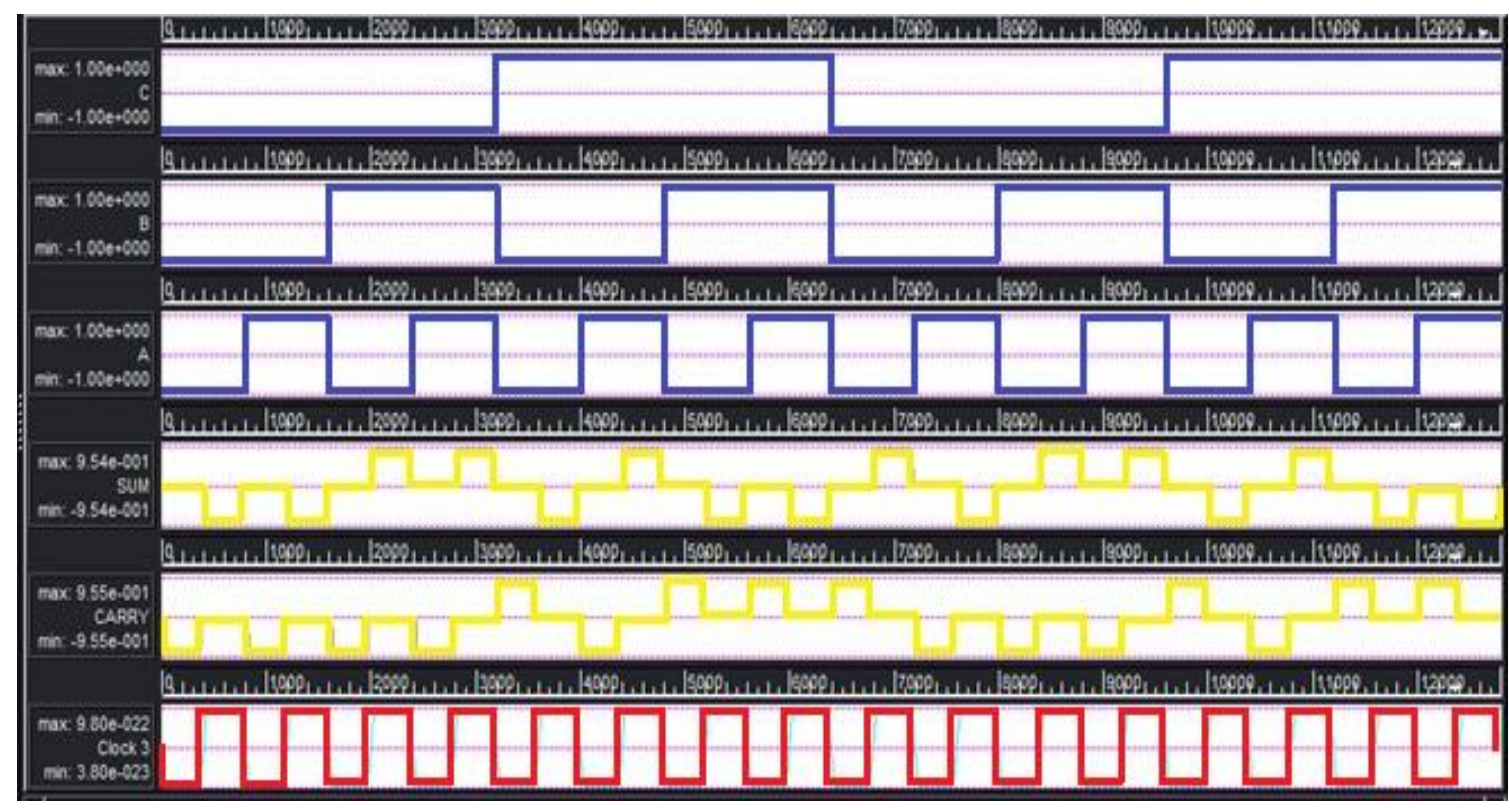

(c)

Figure 9. Proposed full adder, (a) Circuit diagram, (b) QCA implementation, (c) Simulation results of proposed 1-bit full adder

Table 2. Energy dissipation analysis of PFA design for tunneling energies $0.5 \mathrm{E}_{\mathrm{k}}, 1.0 \mathrm{E}_{\mathrm{k}}$ and $1.5 * \mathrm{E}_{\mathrm{k}}$ at $\mathrm{T}=2 \mathrm{~K}$

\begin{tabular}{|c|c|c|c|}
\hline $\begin{array}{l}\text { Tunneling } \\
\text { Energy }\end{array}$ & $\begin{array}{c}\text { Average leakage Energy } \\
\text { Dissipation (meV) }\end{array}$ & $\begin{array}{c}\text { Average Switching Energy } \\
\text { Dissipation }(\mathrm{meV})\end{array}$ & $\begin{array}{c}\text { Average Energy Dissipation of the } \\
\text { circuit (meV) }\end{array}$ \\
\hline $0.5 \mathrm{E}_{\mathrm{k}}$ & 19.62 & 110.35 & 129.97 \\
\hline $1.0 \mathrm{E}_{\mathrm{k}}$ & 57.97 & 96.76 & 154.73 \\
\hline $1.5 \mathrm{E}_{\mathrm{k}}$ & 102.92 & 83.69 & 186.61 \\
\hline
\end{tabular}


Here existing QCA based full adder designs are compared with a new full adder design based upon PMG. The proposed full adder design (PFA) outperforms the existing presented designs in terms of latency and occupational area. The comparison of PFA with the existing designs is presented in Table 3. The PFA design shows $16.6 \%$ enhancement in occupational area and $33.3 \%$ enhancement in latency in comparison to the best design conferred in for single layer as well as multi-layer design [11].

Table 3. Comparison of proposed full adder with the existing layout

\begin{tabular}{ccccc}
\hline Full adder designs & Number of cells & Area $\left(\mu \mathrm{m}^{2}\right)$ & Latency (clock cycles) & Coplanar \\
\hline$[20]$ & 135 & 0.14 & 1.25 & No \\
{$[29]$} & 105 & 0.14 & 0.75 & No \\
{$[31]$} & 95 & 0.08 & 2 & No \\
{$[24]$} & 93 & 0.08 & 1 & No \\
{$[28]$} & 82 & 0.09 & 0.75 & No \\
{$[13]$} & 79 & 0.05 & 1.25 & No \\
{$[30]$} & 79 & 0.06 & 1 & No \\
{$[27]$} & 73 & 0.08 & 0.75 & No \\
{$[8]$} & 73 & 0.04 & 0.75 & No \\
{$[6]$} & 61 & 0.03 & 0.75 & No \\
{$[9]$} & 52 & 0.04 & 0.75 & No \\
{$[13]$} & 51 & 0.03 & 0.75 & No \\
{$[15]$} & 292 & 0.62 & 3.5 & Yes \\
{$[16]$} & 220 & 0.36 & 3 & Yes \\
{$[19]$} & 145 & 0.16 & 1 & Yes \\
{$[25]$} & 105 & 0.17 & 1 & Yes \\
{$[26]$} & 102 & 0.097 & 2 & Yes \\
{$[11]$} & 71 & 0.06 & 1.5 & Yes \\
Proposed & 58 & 0.05 & 1 & Yes \\
\hline
\end{tabular}

\section{COMPARISON OF PMG WITH EXISTING DESIGNS}

The proposed 5-Input coplanar majority gate is verified by a QCADesigner tool. The simulation is done for bistable and coherence vector simulation engine setup for which the total number of samples taken is 32000 with temperature of $2 \mathrm{~K}$. The simulations of PMG are done at the default value of relative permittivity of 12.9 for GaAs and AlGaAs heterostructure implementation. Table 4 depicts the comparison of PMG with the existing designs. It is illustrated that the PMG design occupies an area of $0.01\left(\mu \mathrm{m}^{2}\right)$ which is $50 \%$ less as compared to the best design presented in [22] for single layer as well as multi-layer design.

Table 4. Comparison of PMG with the existing layouts

\begin{tabular}{cccccc}
\hline $\begin{array}{c}\text { QCA Layout } \\
\text { Design in }\end{array}$ & No. of QCA cells & Area $\left(\mu \mathrm{m}^{2}\right)$ & Interference & Single Layer Structure & Multi-Layer Structure \\
\hline$[21]$ & 22 & 0.02 & No & Yes & Yes \\
{$[18]$} & 42 & 0.034 & No & Yes & Yes \\
{$[23]$} & 51 & 0.038 & No & No & Yes \\
Proposed Layout & 17 & 0.01 & No & Yes & Yes \\
\hline
\end{tabular}

\section{CONCLUSION}

In this paper, a novel 5-input coplanar majority gate design with its physical proof is presented. The detailed analysis and energy dissipation of proposed gate were performed. To authenticate the correctness of the proposed gate, a full adder circuit is designed and their power analysis is also done. The results proved that the proposed designs have outgrown all previous mentioned structures and shows remarkable improvement in terms of latency, occupational area, complexity and average energy dissipation. The designs have been verified at three different tunneling energies that is $0.5 \mathrm{Ek}, 1.0 \mathrm{Ek}$ and $1.5 \mathrm{Ek}$ at the temperature of $2 \mathrm{~K}$. The total energy dissipation of the circuit is computed as the sum of average leakage energy and switching energy dissipation. The PMG design has $21.1 \%$ less total energy dissipation than the best reported circuits in at a tunneling energy level of 1.0Ek [22]. 


\section{REFERENCES}

[1] Lent, C.S. and P.D. Tougaw, A device architecture for computing with quantum dots. Proceedings of the IEEE, 1997. 85(4): p. 541-557.

[2] Joachim, C., J. Gimzewski, and A. Aviram, Electronics using hybrid-molecular and mono-molecular devices. Nature, 2000. 408(6812): p. 541.

[3] Bourianoff, G., The future of nanocomputing. Computer, 2003. 36(8): p. 44-53.

[4] Huang, J., M. Momenzadeh, and F. Lombardi, Design of sequential circuits by quantum-dot cellular automata. Microelectronics Journal, 2007. 38(4): p. 525-537.

[5] Walus, K., et al., QCADesigner: A rapid design and simulation tool for quantum-dot cellular automata. IEEE transactions on nanotechnology, 2004. 3(1): p. 26-31.

[6] Navi K, Sayedsalehi S, Farazkish R, Azghadi MR, Five-input majority gate, a new device for quantum-dot cellular automata. Journal of Computational and Theoretical Nanoscience, 2010. 7(8): p. 1546-1553.

[7] Akeela, R. and M.D. Wagh. A five-input majority gate in quantum-dot cellular automata. in NSTI Nanotech. 2011.

[8] Navi, K., et al., A new quantum-dot cellular automata full-adder. Microelectronics Journal, 2010. 41(12): p. $820-826$.

[9] Roohi, A., et al., A symmetric quantum-dot cellular automata design for 5-input majority gate. Journal of Computational Electronics, 2014. 13(3): p. 701-708.

[10] Angizi, S., et al., Design and evaluation of new majority gate-based RAM cell in quantum-dot cellular automata. Microelectron. J., 2015. 46(1): p. 43-51.

[11] Hashemi, S. and K. Navi, A novel robust QCA full-adder. Procedia Materials Science, 2015. 11: p. 376-380.

[12] Sen, B., A. Rajoria, and B.K. Sikdar, Design of efficient full adder in quantum-dot cellular automata. The Scientific World Journal, 2013. 2013.

[13] Hashemi, S., M. Tehrani, and K. Navi, An efficient quantum-dot cellular automata full-adder. Scientific Research and Essays, 2012. 7(2): p. 177-189.

[14] Sheikhfaal, S., et al., Designing efficient QCA logical circuits with power dissipation analysis. Microelectronics Journal, 2015. 46(6): p. 462-471.

[15] Bahar, A.N. and S. Waheed, Design and implementation of an efficient single layer five input majority voter gate in quantum-dot cellular automata. SpringerPlus, 2016. 5(1): p. 636.

[16] Bahar, A.N. and S. Waheed, Design and implementation of an efficient single layer five input majority voter gate in quantum-dot cellular automata. SpringerPlus, 2016. 5(1): p. 636.

[17] Vetteth, A., et al. Quantum-dot cellular automata carry-look-ahead adder and barrel shifter. in IEEE Emerging Telecommunications Technologies Conference. 2002.

[18] Azghadi, M.R., O. Kavehie, and K. Navi, A novel design for quantum-dot cellular automata cells and full adders. arXiv preprint arXiv:1204.2048, 2012.

[19] Farazkish, R. and K. Navi, New efficient five-input majority gate for quantum-dot cellular automata. Journal of Nanoparticle Research, 2012. 14(11): p. 1252.

[20] Zhang, R., et al., A method of majority logic reduction for quantum cellular automata. IEEE Transactions on Nanotechnology, 2004. 3(4): p. 443-450.

[21] Cho, H. and E.E. Swartzlander, Adder designs and analyses for quantum-dot cellular automata. IEEE Transactions on Nanotechnology, 2007. 6(3): p. 374-383.

[22] Mohammadyan, S., S. Angizi, and K. Navi, New fully single layer QCA full-adder cell based on feedback model. International Journal of High Performance Systems Architecture, 2015. 5(4): p. 202-208

[23] Timler, J. and C.S. Lent, Power gain and dissipation in quantum-dot cellular automata. journal of applied physics, 2002. 91(2): p. 823-831.

[24] Farazkish, R., A new quantum-dot cellular automata fault-tolerant five-input majority gate. Journal of nanoparticle research, 2014. 16(2): p. 2259.

[25] Zhang, R., et al. Performance comparison of quantum-dot cellular automata adders. in Circuits and Systems, 2005. ISCAS 2005. IEEE International Symposium on. 2005. IEEE.

[26] Wang, W., K. Walus, and G.A. Jullien. Quantum-dot cellular automata adders. in Nanotechnology, 2003. IEEENANO 2003. 2003 Third IEEE Conference on. 2003. IEEE.

[27] Hänninen, I. and J. Takala, Binary adders on quantum-dot cellular automata. Journal of Signal Processing Systems, 2010. 58(1): p. 87-103.

[28] Sayedsalehi, S., et al., Restoring and non-restoring array divider designs in quantum-dot cellular automata. Information sciences, 2015. 311: p. 86-101.

[29] Abdullah-Al-Shafi, M. and A.N. Bahar, Optimized design and performance analysis of novel comparator and full adder in nanoscale. Cogent Engineering, 2016. 3(1): p. 1237864.

[30] Cho, H. and E.E. Swartzlander Jr, Adder and multiplier design in quantum-dot cellular automata. IEEE Transactions on Computers, 2009. 58(6): p. 721-727.

[31] Cho, H., Adder and multiplier design and analysis in quantum-dot cellular automata. 2006.

[32] Abdullah-Al-Shafi, M., et al., Performance evaluation of efficient combinational logic design using nanomaterial electronics. Cogent Engineering, 2017. 4(1): p. 1349539.

[33] Abdullah-Al-Shafi, M., et al., Power analysis dataset for QCA based multiplexer circuits. Data in brief, 2017. 11: p. 593.

[34] Shamsabadi, A.S., et al., Applying inherent capabilities of quantum-dot cellular automata to design: D flip-flop case study. Journal of systems architecture, 2009. 55(3): p. 180-187. 
[35] Vankamamidi, V., M. Ottavi, and F. Lombardi, A serial memory by quantum-dot cellular automata (QCA). IEEE Transactions on Computers, 2008. 57(5): p. 606-618

[36] Vetteth, A., et al., Quantum-dot cellular automata of flip-flops. ATIPS Laboratory, 2003. 2500.

[37] Yang, X., L. Cai, and X. Zhao, Low power dual-edge triggered flip-flop structure in quantum dot cellular automata. Electronics letters, 2010. 46(12): p. 825-826.

[38] Hashemi, S. and K. Navi, New robust QCA D flip flop and memory structures. Microelectronics Journal, 2012. 43(12): p. 929-940.

[39] Abdullah-Al-Shafi, M. and A.N. Bahar, Ultra-efficient design of robust RS flip-flop in nanoscale with energy dissipation study. Cogent Engineering, 2017. 4(1): p. 1391060.

[40] Abdullah-Al-Shafi, M., et al., Designing single layer counter in quantum-dot cellular automata with energy dissipation analysis. Ain Shams Engineering Journal, 2017.

[41] Bahar, A.N., et al., Energy dissipation dataset for reversible logic gates in quantum dot-cellular automata. Data in brief, 2017. 10: p. 557-560.

[42] Dysart, T.J. and P.M. Kogge. Probabilistic analysis of a molecular quantum-dot cellular automata adder. in Defect and Fault-Tolerance in VLSI Systems, 2007. DFT'07. 22nd IEEE International Symposium on. 2007. IEEE.

[43] Crocker, M., et al., Molecular QCA design with chemically reasonable constraints. ACM Journal on Emerging Technologies in Computing Systems (JETC), 2008. 4(2): p. 9.

[44] Srivastava, S., S. Sarkar, and S. Bhanja, Estimation of upper bound of power dissipation in QCA circuits. IEEE transactions on nanotechnology, 2009. 8(1): p. 116-127. 